9. Ostriker, J. Ann. N.Y. Acad. Sci. 302, 229-243 (1977)

10. Bevington, P. Data Reduction and Error Analysis for the Physical Sciences (McGraw-Hill, New York, 1978)

11. Clark, G., Markert, T. \& Li, F. Astrophys. J. Lett. 199, L93-L96 (1975)

12. Grindlay, J. et al. Astrophys. J. Lett. 205, L127-L130 (1976).

13. Clark, G, et al. Mon. Not. R. astr. Soc. 179, 651-658 (1977)

14. McClintock, J. et al. Nature 270, 320-321 (1977).

15. McClintock, J., Canizares, C. \& Backman, D. Astrophys. J. Lett. 223, L75-L78 (1978)

16. Grindlay, J. et al. Nature 274, 567-568 (1978).

16. Grindlay, J. et al. Nature 274, 567-568 (1978).
17. Lewin, W. COSPAR Ming., Innsbruck (1978).

17. Lewin, W. COSPAR Ming.,
18. Milgrom, M. preprint (1978).

19. van den Heuvel, E. Ann. N.Y. Acad. Sci. 302, 14-35 (1977)

\section{Definition of a black hole}

TIPLER has given a new definition for a black hole ${ }^{1}$. These objects had previously been properly defined only in asymptotically flat spacetimes ${ }^{2}$, but it seems unlikely that our Universe is one of these. One of the well known properties of a black hole, proved in asymptotically flat spacetimes, is that its 'surface area' can never decrease. By means of this new definition Tipler has shown that this area theorem may well not be true in our Universe. However, Tipler's definition is complicated, and it is not obvious that it corresponds to our usual concept of a black hole. I show here that it is equivalent to a definition that is both simpler and more clearly a formalisation of what we mean by a black hole.

I use the notation of refs 1 and 2 . Let $T$ be the set of points of a stably causal spacetime which belong to non-cosmological trapped surfaces. Tipler defines a black hole as the closure of the topologically smallest future set $I^{+}$such that $I^{+}$contains $T$, and the boundary of $\mathrm{I}^{+}$is generated by (future inextensible) null geodesic segments which are boundary generators of TIPs.

We would like a black hole to contain $T$. It should not be possible to escape from a black hole, so the hole should also contain $I^{+}(T)$. Furthermore, any points from which it is impossible to avoid falling into a black hole might as well be said to be within that hole. That is, a black hole should be the closure of $B=D^{-}\left(I^{+}(T)\right)$; I shall show that $B$ satisfies the conditions set out by Tipler for $I^{+}$.

$B$ is a future set: suppose $x \in B$ and $y \in I^{+}(x)$. There is a future directed timelike curve from $x$ to $y$. If it meets $I^{+}(T)$ then $y \in I^{+}(T)$, so $y \in B$. If it does not meet $I^{+}(T)$, it must do so when extended along any future directed non-spacelike curve $\alpha$ from $y$ as $x \in D^{-}\left(I^{+}(T)\right)$. Therefore, any such $\alpha$ meets $I^{+}(T)$, so $y \in B$.

The boundary of $B$ is generated by null geodesic segments which are boundary generators of TIPs: let $x$ be on the boundary of $B$. Let $\left(x_{\mathrm{i}}\right)$ be a sequence of points lying to the past of $x$, not in $B$, with $x$ as their limit point. There is a future inextensible non-spacelike curve $\gamma_{\mathrm{i}}$ from each $x_{\mathrm{i}}$ that does not enter $I^{+}(T)$. By proposition 6.2.1. of ref. 2 there is a future inextensible non-spacelike curve $\gamma$ from $x$ which is a limit curve for the $\gamma_{i}$.

As $\bar{B}$ is a closed future set $\gamma$ can never leave it. As no $\gamma_{\mathrm{i}}$ can enter $B, \gamma$ cannot enter the interior of $B$. But $I^{+}(\gamma) \subset B$, so $\gamma$ cannot enter its own future. Therefore, it must be a null geodesic.

$\bar{I}(\gamma)$ is a TIP (see ref. 3). As $\gamma$ cannot enter its own future it cannot enter its own past, $I^{-}(\gamma)$. Therefore, it is a boundary generator of $I^{-}(\gamma)$.

It remains to be proved that $B$ is the smallest such $I^{+}$. It has been shown that from any point on the boundary of $B$ there is a future directed null geodesic $\gamma$ that does not enter the interior of $B$, so cannot enter $I^{+}(T)$. Therefore, the boundary of $B$ is not part of $B$, and $B$ is open.

Suppose there is an $I^{+}$that is contained in $B$ but not equal to it. We can then find a point $p$ which is in $B$ but not in $I^{+}$. Let $\alpha$ be a future directed timelike line from $p$. It must meet $I^{+}(T)$, and hence must enter $I^{+}$. Let it cross the boundary of $I^{+}$at $y$. There is a future directed null geodesic generator $\gamma$ of the boundary of $I^{+}$ from $y$. It is future inextensible, and cannot enter $I^{+}(T)$ as otherwise it would be in the interior of $I^{+}$. Thus, by proceeding from $p$ along $\alpha$ to $y$ then along $\gamma$, we can construct a future inextensible non-spacelike curve from $p$ which does not meet $I^{+}(T)$. This contradicts $p \in B$.
Therefore $B$ is the smallest such $I^{+}$, and the black hole defined by Tipler is exactly $\bar{D}^{-}\left(I^{+}(T)\right)$.

Department of Mathematics,

C. W. LEE

Wigan College of Technology,

Parsons Walk, Wigan,

Greater Manchester, UK

Received 4 July; accepted 18 September 1978

1. Tipler, F. J. Nature 270, 500 (1977),

2. Hawking, S. W. \& Ellis, G. F. R. The Large Scale Structure of Space-Time (Cambridge University Press, 1973)

3. Geroch, R. P., Kronheimer, E. H. \& Penrose R. Proc. R. Soc. A327, 545 (1972).

\section{Neutrinos from binary pulsars}

PULSARS are believed to produce high energy particles. If high energy protons produced by the pulsar strike a target, say a binary companion, high energy neutrinos are produced in the collisions. At the same time, schemes for detecting high energy neutrinos produced in nature are currently being considered (refs 1-4 and K. Lande, personal communication). Here we point out that binary systems containing moderately young pulsars (ages $T<10^{4} \mathrm{yr}$, that is comparable to NP0532) may emit high energy neutrinos $\left(10^{12}<E_{\gamma}<10^{14} \mathrm{eV}\right)$ at detectable levels. Such pulsars are assumed to have total luminosities of the order $10^{38} \mathrm{erg} \mathrm{s}^{-1}$. Discussion of feasible neutrino telescopes (such as / the deep underwater muon and neutrino detector, DUMAND ${ }^{1-4}$ ) suggests that fluxes from point sources as small as $10^{2}-1 \mathrm{eV} \mathrm{cm}^{-2} \mathrm{~s}^{-1}$ in high energy neutrinos may eventually be detectable. Neutrino detectors on a more modest scale, such as that of Lande (personal communication) will be operating within $1-3 \mathrm{yr}$, and have detection thresholds of $10^{5} \mathrm{eV} \mathrm{cm}^{-2} \mathrm{~s}^{-1}$. Thus, a pulsar emitting $3 \times 10^{38} \mathrm{erg} \mathrm{s}^{-1}$ in high energy neutrinos at $3 \mathrm{kpc}$ would be detectable even with Lande's detector, and the DUMAND detector may be able to detect sources that are weaker by three to five orders of magnitude.

We assume that at least some of the pulsars emit a fair fraction $(\Im 1 \%$ ) of their energy in the form of high energy protons $\left(10^{13}<E_{\mathrm{p}}<10^{16} \mathrm{eV}\right)$. This energy range is consistent with the upper limit ${ }^{5}$ of the potential near the star

$$
V_{\max }=10^{16}\left(B / 10^{12} \mathrm{G}\right)\left(\Omega / 200 \mathrm{~s}^{-1}\right)^{2} V
$$

where $B$ is the magnitude of the magnetic field at the stellar surface, and $\Omega$ is the frequency of rotation. That young pulsars can produce very energetic particles is implied by the very energetic $\left(E_{\gamma} \approx 10^{12} \mathrm{eV}\right)$ pulsed $\gamma$ rays $^{6}$ emitted by NP0532. The energy in the protons is converted efficiently to high energy neutrinos if either the protons traverse more than $\sim 50 \mathrm{~g}$ of material, or they traverse a column density of $10^{28}$ photons $\mathrm{cm}^{-2}$ in photons that are not too far above the pion production threshold in the rest frame of the protons. In each case, the protons are stopped by pion and kaon producing processes, and the charged mesons that are produced decay into three neutrinos and an electron. Roughly half the energy in the original proton goes into high energy neutrinos, which typically have energies of the order $10^{-2} E_{\mathrm{p}}$ to $10^{-1} E_{\mathrm{p}}$ (ref. 3).

There are several plausible ways in which the high energy protons might encounter enough target material to convert $1 \%$ to $50 \%$ of their energy to neutrinos.

If the companion star fills its Roche lobe, it could occupy nearly half the sky as seen from the pulsar, thus blocking a proportionate fraction of the high energy protons. Moreover, the overflowing material may completely engulf the pulsar.

Accreting matter can pile up at the magnetopause (which in the case of a pulsar would be made up of very low frequency electromagnetic Alfv́en waves). It has been suggested ${ }^{7,8}$ that such a pile up occurs in several intermediate and rapidly rotating binary $\mathrm{X}$-ray sources. 